\title{
Tratamiento del ardor bucal y de la boca seca. Nuevas tendencias
}

\section{Treatment of burning mouth. New trends}

\author{
De Luca Monasterios F*, Rodríguez de Rivera Campillo ME**
}

\begin{abstract}
RESUMEN
En la presente revisión, abordaremos el tratamiento del SBA. Dado que desconocemos con exactitud la etiopatogenia, el tratamiento suele orientarse hacia el manejo de los síntomas. Se pretende: Tratar los procesos relacionados con ardor bucal eliminando los factores locales o irritantes (tabaco, alcohol, comidas picantes y bebidas ácidas, aristas cortantes, xerostomía, disgeusia, parafunciones, bruxismo, mordisqueo o succión de mucosas, hábitos parafuncionales orales, irritación mecánica, prótesis desajustadas o alergia a materiales dentales) ya que estos pueden aumentan el ardor. Prevenir y tratar alteraciones o lesiones sobre la mucosa bucal. Por otro lado se deben tratar y/o remitir procesos psicopatológicos. El hecho de escuchar con atención al paciente y explicarle el motivo de sus molestias suele calmar al paciente y disminuir los síntomas por lo que se aconseja una evaluación de forma periódica cada 6 meses. El tratamiento se basa en cuatro posibilidades terapéuticas: Odontológico: local-conservador e higiénico (pulir-redondear cúspides o aristas cortantes; realizar tratamiento protésico, la utilización de barreras físicas como geles o protectores dentales); Farmacológicos, Psicológicos: la depresión y la ansiedad desempeñan un papel importante en la modulación de la percepción del dolor, se han evidenciado en estos pacientes alteraciones del sistema nervioso central y del sistema nervioso periférico por lo que el uso de fármacos psicoactivos antidepresivos se han justificado, entre ellos el clonacepam tópico (Rivotril@), con una mejoría del 50\% de los síntomas. Dados los escasos resultados obtenidos en el tratamiento del cuadro, se han propuesto una serie de tratamientos Alternativos para intentar controlarlo, los cuales han tenido aparentes resultados beneficiosos: infrarrojos, el láser de baja intensidad aplicado en la zona referida por el paciente, la fitoterapia con efectos analgésicos y antidepresivos, con mejoría del 52\% de los pacientes, el aloe vera, la acupuntura y el tratamiento cognitivo-conductual que ayuda a reducir la ansiedad. Teniendo en cuenta que ninguno de los tratamientos es definitivo ni universal, podemos concluir que existen tratamientos eficaces para algunos de los casos, pero en otros muchos se sigue buscando un procedimiento que resulte eficaz.

Palabras clave: Irritantes orales, tratamiento ardor bucal, síndrome de boca ardiente, clonacepam.
\end{abstract}

\section{SUMMARY}

In this review will tackle treatment SBA. Because the exact etiopathogenesis is unknown, treatment is directed toward symptom management. The aim is: Treat the processes associated with burning mouth or eliminating local irritants (snuff, alcohol, spicy foods and acidic beverages, cutting edges, xerostomia, dysgeusia, parafunctions, bruxism, chewing or sucking mucous, oral parafunctional habits, mechanical irritation, unadjusted dentures or allergies to dental materials) as these can increase the burning. Prevent and treat disease or disorder of the oral mucosa. On the other hand should be treated and/or remit psychopathological processes. Just listen carefully to the patient and explain the reason for their discomfort usually calm the patient and reduce the symptoms so a periodic assessment every 6 months is advised. The treatment is based on four therapeutic possibilities: Dental: locally conservative and hygienic (polish-rounded cusps or sharp edges; make prosthetic treatment, the use of physical barriers such as gels or dental dams); Pharmacological, Psychological: depression and anxiety play an important role in modulating pain perception, have been demonstrated in these patients alterations of the central

* $\quad$ DDS, Máster en Medicina Bucal. Facultad de Odontología. Universidad de Barcelona.

** PhD, DDS, MD. Dermatóloga. Odontóloga. Profesora Asociada Medicina Bucal. Facultad de Odontología. Universidad de Barcelona. 
nervous system and the peripheral nervous system so that the use of antidepressants have been justified psychoactive drugs, including topical clonazepam (Rivotril $($ ) ) with a $50 \%$ improvement in symptoms. Given the poor results in the treatment of the condition have been proposed a number of Alternative treatments to try to control it, which have apparent beneficial results: infrared, low level laser applied in the area reported by the patient, with herbal medicine analgesic and antidepressant effects, with improvement in $52 \%$ of patients, aloe vera, acupuncture and cognitivebehavioral treatment to help reduce anxiety. Given that neither treatment is definitive or universal, we can conclude that there are effective treatments some cases, but many others are still looking for a method to be effective.

Key words: Oral irritants, treatment burning mouth, burning mouth syndrome clonazepam.

Fecha de recepción: 15 de enero de 2014.

Aceptado para publicación: 19 de marzo de 2014.

De Luca Monasterios F, Rodríguez de Rivera Campillo ME. Tratamiento del ardor bucal y de la boca seca. Nuevas tendencias. Av. Odontoestomatol 2014; 30 (3): 139-143.

\section{TRATAMIENTO LOCAL-CONSERVADOR}

Deben eliminarse los factores locales que aumentan la sensación de boca ardiente, como tabaco, alcohol, comidas picantes y bebidas ácidas, que irriten la mucosa oral, aristas cortantes, xerostomía, disgeusia, parafunciones, bruxismo, mordisqueo o succión de mucosas. También es necesario investigar la presencia de hábitos parafuncionales orales, irritación mecánica, prótesis desajustadas o alergia a componentes de materiales dentales $(3,4)$. La eliminación de todos estos factores suele traducirse en una mejoría clínica. De forma genérica y teniendo en cuenta que ninguno de los tratamientos es definitivo ni universal, se propone que, en la mayoría de los casos, se deba $(1,2)$ :

1. Escuchar de forma atenta y pacientemente al enfermo.

2. Explicarle de forma sencilla el motivo de sus molestias.

3. Plantear estrategia terapéutica.

4. Eliminar irritantes.

5. Tratamiento odontológico: local-conservador e higiénico.

6. Pulir-redondear cúspides o aristas.

7. Realizar tratamiento protésico (dientes ausentes).

8. Barreras físicas: geles, protectores dentales.

Hay diferentes claves terapéuticas recomendadas del síndrome de boca ardiente basadas en la experiencia clínica (Figura 1) (1).

Es importante el tiempo de evolución del SBA, cuando el paciente acude a la consulta. Recién instaura- do el cuadro, el tratamiento conservador tiende a ser más eficaz. El objetivo será eliminar o atenuar la agresión local, puliendo dientes o superficies irregulares de prótesis, aplicando productos lubricantes de cierta densidad y propiedades adecuadas, o bien con aparatos (férulas, placas de descarga, protectores plásticos); solos o en combinación (5). También se deben evitar los hábitos tóxicos (tabaco, alcohol), que contribuyen a resecar e irritar la mucosa oral. Factores sistémicos como infecciones subagudas o crónicas (3) (candidiasis, hábitos alimentarios inadecuados y deshidratación —absoluta o relativa) requerirán un estudio analítico. No es infrecuente descubrir alteraciones en el metabolismo del hierro, que hacen recomendable investigar su origen e introducir una compensación alimentaria. Por otra parte los factores psicológicos son difíciles de controlar. De hecho, a menudo estos pacientes ya están recibiendo tratamiento farmacológico ansiolítico o antidepresivo, sin que ello haya evitado el desarrollo de la parafunción, como parte fundamental del SBA que presentan. Por tanto, lo más recomendable no es exceder en la administración de fármacos, sino orientar al paciente en otro sentido. Se debe recomendar por ejemplo la ingesta de infusiones relajantes y, por el contrario, evitar bebidas excitantes $(1,6,7)$.

En función de la edad y las capacidades del paciente, es recomendable que practique ejercicio físico aeróbico de forma regular, mejor si lo hace fuera de su entorno habitual. De esa manera aumentan las posibilidades de evadirse, aunque sea temporalmen- 


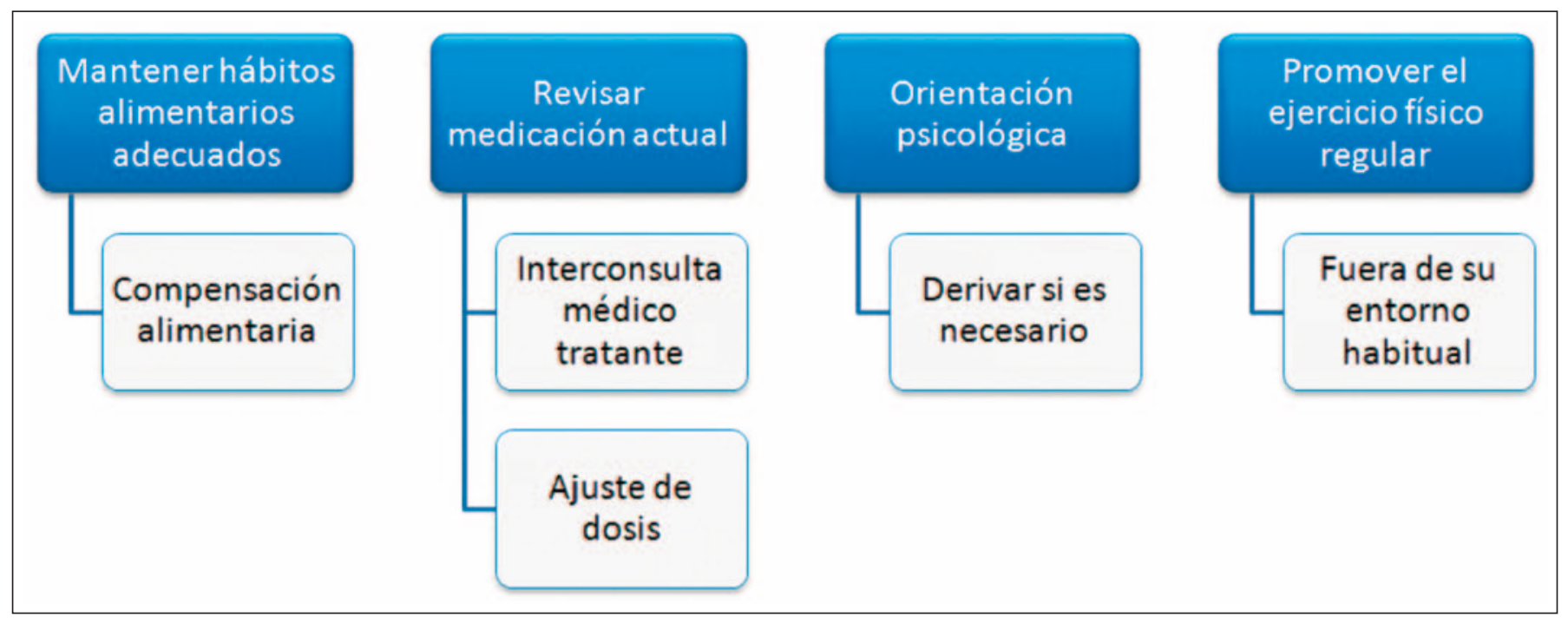

Fig. 1. Claves terapéuticas del SBA.

te, de problemas cotidianos, y de relacionarse con otras personas. De esta manera se puede conseguir una mayor estabilidad emocional y ayuda a regular los mediadores de la inflamación y del dolor produciendo endorfinas. En caso de que la combinación de estas medidas no proporcione el efecto esperado, se puede recurrir a tratamiento farmacológico (1).

Es muy importante explicar a paciente la naturaleza de su patología y la ausencia de malignidad, así se logra disminuir la ansiedad y mejorar considerablemente la sintomatología. Es de gran ayuda el uso de una escala visual analógica que permita cuantificar las molestias.

\section{FARMACOTERAPIA}

Aunque la etiopatogenia del SBA no es bien conocida, problemas como la depresión y la ansiedad parecen desempeñar un papel importante en la modulación de la percepción del dolor (8). Por ello es frecuente orientar el tratamiento hacia fármacos antidepresivos en estos pacientes. Por otra parte, la identificación de alteraciones sensoriales evidencia una base biológica relacionada con alteraciones del sistema nervioso central y del sistema nervioso periférico. Esta teoría parece reforzar la acción beneficiosa del clonacepam $(6,9)$.
Los cambios en la tolerancia al calor, la disgeusia y el aumento de la excitabilidad del reflejo palpebral que tienden a presentar estos pacientes están relacionados con la disfunción del sistema dopaminérgico a nivel central. Esta disfunción justificaría la administración sistémica de fármacos antiepilépticos como gabapentina y clonacepam (1). Por otra parte, se ha observado que el SBA presenta una serie de características comunes con otros síndromes dolorosos crónicos, justificando la administración de fármacos como capsaicina y bencidaminas (1). La amisulprida (antagonista selectivo de la dopamina) parece haber dado buenos resultados en el tratamiento de SBA a corto plazo, si bien, según los autores, conviene ampliar el estudio a largo plazo (8).

Otros múltiples fármacos psicoactivos, con resultado variables, han sido utilizados tratamiento del cuadro (Tabla 1). De entre todos ellos podemos destacar los resultados obtenidos con la amisulprida y con el clonacepam tópico (Rivotril $\left.{ }^{\circledR}\right)$, en el caso del clonacepam se publican resultados de una mejoría de hasta el $50 \%(6,7,9)$.

\section{MÉTODOS ALTERNATIVOS}

Dados los escasos resultados obtenidos en el tratamiento del cuadro, se han propuesto múltiples tratamientos alternativos para intentar controlar este cua- 


\begin{tabular}{|c|c|c|}
\hline \multicolumn{3}{|c|}{$\begin{array}{c}\text { TABLA 1.- TRATAMIENTOS UTILIZADOS } \\
\text { ACTUALMENTE EN PACIENTES CON } \\
\text { ARDOR BUCAL }\end{array}$} \\
\hline $\begin{array}{l}\text { Tratamiento } \\
\text { sustitutivo }\end{array}$ & $\begin{array}{c}\text { Fármacos } \\
\text { psicoactivos }\end{array}$ & $\begin{array}{l}\text { Métodos } \\
\text { alternativos }\end{array}$ \\
\hline $\begin{array}{l}\text { - Hormonal } \\
\text { - Vitaminas B1, } \\
\text { B2 y B6 } \\
\text { - Sialogogos } \\
\text { - Corticoides } \\
\text { - Ácido alfa lipoico } \\
\text { - Psicoterapia } \\
\text { - Psicoterapia + } \\
\text { antidepresivos }\end{array}$ & $\begin{array}{l}\text { - Antidepresivos } \\
\text { - Benzodiacepinas } \\
\text { - Clonacepam } \\
\text { - Gabapentina } \\
\text { - Capsaicina } \\
\text { - Benzidaminas } \\
\text { - Clorhidrato de } \\
\text { benzidamina } \\
\text { - Amisulprida }\end{array}$ & $\begin{array}{l}\text { - Anestésicos } \\
\text { locales } \\
\text { - Láser baja } \\
\text { intensidad } \\
\text { - Acupuntura } \\
\text { - Hipnosis } \\
\text { - Psicoterapia } \\
\text { - Fitoterapia } \\
\text { - Aloe vera }\end{array}$ \\
\hline
\end{tabular}

dro (Tabla 1). Autores japoneses relacionan el SBA con la función del sistema nervioso autónomo e irradiando con infrarrojos el núcleo estrellado observaron una mejoría semiológica en pacientes afectados (10). También se ha propuesto el láser de baja intensidad, aplicado en la zona referida por el paciente, con aparentes resultados beneficiosos (11). La fitoterapia ha sido eficaz, con efectos analgésicos y antidepresivos, con mejoría significativa de hasta el 52\% de los pacientes con SBA, manteniéndose el efecto durante 12 semanas en un $51 \%$ de los pacientes (12). La acupuntura también se considera un método alternativo para el tratamiento del dolor (13). Plantas como aloe vera, en combinación con un protector lingual, también parecen contribuyen a minimizar los traumas repetitivos de la mucosa oral (5).

El tratamiento cognitivo-conductual ayuda a reducir la ansiedad, con lo que disminuye también significativamente el dolor en estos pacientes (14). Para algunos autores, una pequeña proporción de casos presenta remisión espontánea durante los 5 primeros años de tratamiento; en un $42 \%$ mejoran los síntomas significativamente, y esta mejora alcanza un $60 \%$ si se asocian clonacepam y psicoterapia.

Finalmente podemos concluir que se encuentran tratamientos eficaces para algunos casos, pero en otros muchos se sigue buscando un procedimiento que resulte eficaz.

\section{BIBLIOGRAFÍA}

1. Chimenos-Küstner E, Arcos-Guerra C, MarquesSoares M. Síndrome de boca ardiente: claves diagnósticas y terapéuticas. Med Clin (Barc) 2013; pii: S0025-7753(13)00736-7. doi: 10.1016/ j.medcli.2013.09.027. [Epub ahead of print].

2. Protocolos de Medicina Bucal, Sociedad española de Medicina Oral. Síndrome de Boca Ardiente. Disponible: http://www.semo.es/. Accedido en 14 de enero de 2013.

3. López-Jornet P, Camacho-Alonso F, Andújar-Mateos P, Sánchez-Siles M, Gómez-García F. Burning mouth syndrome: Update. Med Oral Patol Oral Cir Bucal 2010;15:e562-8.

4. Fuertes A, González López B, Gamell M, Girons J, López López J, Chimenos E, Jané Sala E. Alergia a las prótesis dentales con resinas. Revisión del problema. DENTUM 2009;9:6-14.

5. López-Jornet P, Camacho-Alonso F, Molino-Pagan D. Prospective, randomized, double-blind, clinical evaluation of Aloe vera Barbadensis, applied in combination with a tongue protector to treat burning mouth syndrome. J Oral Pathol Med 2013;42:295-301.

6. Rodríguez de Rivera Campillo E, López-L J, Chimenos-Küstner E. Response to topical clonazepam in patients with burning mouth syndrome: A clinical study. Bull Group Int Rech Sci Stomatol Odontol 2010;49:19-29.

7. Rodríguez de Rivera-Campillo ME, López-López J, Chimenos-Küstner E. Tratamiento del síndrome de boca ardiente con clonacepam tópico. Piel 2011;26:263-8.

8. Zakrzewska JM. Multi-dimensionality of chronic pain of the oral cavity and face. J Headache Pain 2013;14:37.

9. Rodríguez de Rivera-Camnpillo ME, López-López $\mathrm{J}$. Evaluation of the response to treatment and clinical evolution in patients with burning mouth syndrome. Med Oral Patol Oral Cir Bucal. 2013; 18:e403-10. 
10. Rodríguez-Cerdeira C, Sánchez-Blanco E. Treatment of burning mouth syndrome with amisulpride. J Clin Med Res 2012;4:167-71.

11. Momota Y, Takano H, Kani K, Matsumoto F, Motegi K, Aota K, et al. Frequency analysis of heart rate variability: A useful assessment tool of linearly polarized near infrared irradiation to stellate ganglion area for burning mouth syndrome. Pain Med 2013;14:351-7.

12. Spanemberg JC, Cherubini K, de Figueiredo MA, Gomes AP, Campos MM, Salum FG, et al Effect of an herbal compound for treatment of burning mouth syndrome: randomized, controlled, double-blind clinical trial. Oral Surg Oral Med Oral Pathol Oral Radiol 2012;113: 373-7.

13. Sardella A, Lodi G, Tarozzi M, Varoni E, Franchini R, Carrassi A. Acupuncture and Burning Mouth
Syndrome: A Pilot Study.Pain Pract. 2013;13:62732.

14. Komiyama O, Nishimura H, Makiyama T, lida T, Obara R, Shinoda M, et al. Group cognitivebehavioral intervention for patients with burning mouth syndrome. J Oral Sci 2013;55:17-22.

\section{CORRESPONDENCIA}

Dra. María Eugenia Rodríguez de Rivera Campillo Campus Universitario de Bellvitge Departamento de Odontoestomatología Facultad de Odontología

Pabellón de Gobierno

Feixa Llarga, s/n

08907 L'Hospitalet de Llobregat. Barcelona

Correo electrónico: 20254err@gmail.com 